\section{Use of a New Imaging Technique to Document Deformations Recorded in the Environmental Scanning Electron Microscope}

\author{
Edward F. O'Neil," Hamlin M. Jennings, ${ }^{* \star}$ and Jeffrey J. Thomas ${ }^{* *}$ \\ ${ }^{*}$ US Army Corps of Engineers, ${ }^{* *}$ Northwestern University \\ Ed.F.O'Neil@erdc.usace.army.mil
}

The introduction of the environmental scanning electron microscope (ESEM) greatly improved the functionality of scanning electron microscope (SEM) technology by allowing objects to be imaged in water-vapor, under low vacuum, and without the need for coating to facilitate electrical conductivity. The low vacuum environment allows imaging of delicate structures, such as needlelike crystals, without fear of damaging the object in the process of viewing it. The elimination of metallic coating allows the microscopist to view specimens in a more natural environment, such that the specimen can change with respect to time. The latter condition is very valuable to scientists and engineers studying phenomena that are time dependent, such as environmental expansion and contraction or static and dynamic fracture. Two areas where the functionality of the ESEM is valuable to the US Army Corps of Engineers is in studying damage done to cement paste from cycles of freezing and thawing, and the deformation associated with the development of cracks in concrete under the influences of static or dynamic loading.

The Concrete and Materials Branch of the Geotechnical and Structures Laboratory at the Engineer Research and Development Center in Vicksburg, Mississippi is the Corps of Engineers' primary facility for the study of cement and concrete materials for both civil works and military applications. Research conducted here ranges from development of new concrete materials for blast-resistant, structural fortifications to study of the mechanisms within cement paste that bind concrete together or cause its destruction. The microscopic end of this broad research area involves many uses of the ESEM. The two areas mentioned above involve the use of the microscope, collection of time-dependent imagery data (in both cases strain-induced deformation), and processing of these data with deformation mapping technology (DMT) developed at Northwestern University [ref. $1-3]$ to produce maps that document deformational changes in the specimen resulting from a straining event. In the aforementioned cases, the straining events are internal deformation from the forces of expanding ice during freezing and thawing cycles, and deformation in front of a crack that is propagating due to external loading to the specimen.

The DMT is the key to capturing the changes that occur during the straining event since such events can result in very small deformations. Because the ESEM can capture digital images at any time, two images can be taken that bracket the deformation in the straining event: one before the event (the reference image), and one after (the deformed image). Both images are essentially arrays of 8-bit digital data containing the gray-scale intensities of each pixel in their respective images. Because of the deformation caused by the straining event, there will be small differences in the relative position of individual microstructural features in the two images. The DMT compares the two images and calculates the deformation of each pixel. This is accomplished by selecting a 20 by 20 pixel target window surrounding the first pixel of interest in the deformed image, and superimposing that window on a 40 by 40 pixel window in the reference image. The intensity of each pixel in the 20 by 20 target window is then compared to the corresponding pixel in the reference window and a summation of the squares of the differences in intensities between each pixel pair is recorded. The target window then is moved one pixel either horizontally

or vertically on the reference window and the summation process repeated. This procedure is continued until the reference window has been completely searched. The match for the location of the first deformed image pixel is determined by the least-squares summation with the smallest magnitude. A new pair of target and reference windows is created by moving the 20 by 20 pixel target window to a new pixel of interest and the location of this pixel is determined by the least-squares summation procedure. The entire process is repeated until every pixel in the deformed image has received a deformation location.

The result is an array of magnitudes of the deformations of each pixel from the before event to the after event. This array is post-processed to visually describe the deformations. The post-processing software assigns a color code to each pixel that is representative of its type and magnitude of deformation. Two types of deformation are distinguished. Pixels that are found to have moved closer to each other are referred to as compactions and are colored with two shades of blue to represent their magnitude (light blue for moderate and dark blue for large compactions). Pixels that are found to move away from each other are referred to as rarefactions and are colored with two shades of red (light red for moderate rarefactions and dark red for large). A third color, white, is used to represent very light magnitudes of both rarefactions and compactions. The output deformation map is a color representation of the deformations that took place during the straining event.

Figures 1 and 2 illustrate the color coding assigned by the DMT postprocessing. These figures are deformation maps created by comparing ESEM

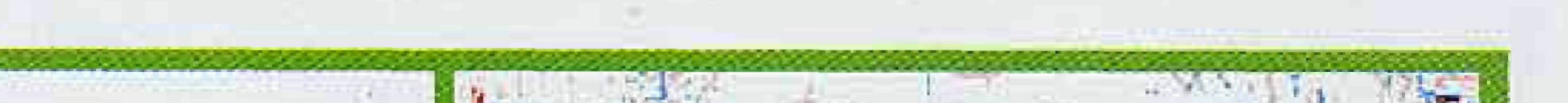

Figure 1. Deformation map of 1 cycle of freezing and thawing.
Figure 2. Deformation map of 3 cycles of freezing and thawing.

images of cement paste taken before freezing and after 1 and 3 cycles of freezing and thawing respectively. A $2 \mu \mathrm{m}$ thick water-saturated specimen of cement paste was placed on a cold stage and imaged at $250 \mathrm{X}$ magnification in an Electroscan 2020 ESEM. Figure 3 shows the photomicrograph of the imaged area. The before event image was taken at $+5^{\circ} \mathrm{C}$ before the specimen was frozen. The specimen then was taken to $-20^{\circ} \mathrm{C}$, held for 30 minutes, and returned to $+5^{\circ} \mathrm{C}$ where the first after-event image was made representing the deformed shape after 1 cycle of freezing and thawing. Two more cycles of freezing to $-20^{\circ} \mathrm{C}$ were applied to the specimen and the specimen returned to $+5^{\circ} \mathrm{C}$ for the second after-event image to be taken. Figure 1 is almost entirely white, indicating only small magnitudes of either rarefaction or compaction apply to the vast majority of pixels in this comparison of images before freezing and after 1 cycle. Some moderate and heavy rarefactions can be seen in the upper left and lower right corners of this deformation map. The data imply that very little damage was done to the cement paste on the first freezing cycle. Figure 2 shows greater damage at both the upper left and lower right corners of the image, as well as more damage in general throughout the specimen. These data imply that the specimen was damaged to a greater extent by the three cycles of freezing and thawing. The most dramatic evidence of deformation is seen in the lower right corner of figure 2 where a ring of heavy rarefaction surrounds an area of moderate and heavy compaction. Some damage is seen 


\section{Quantax}

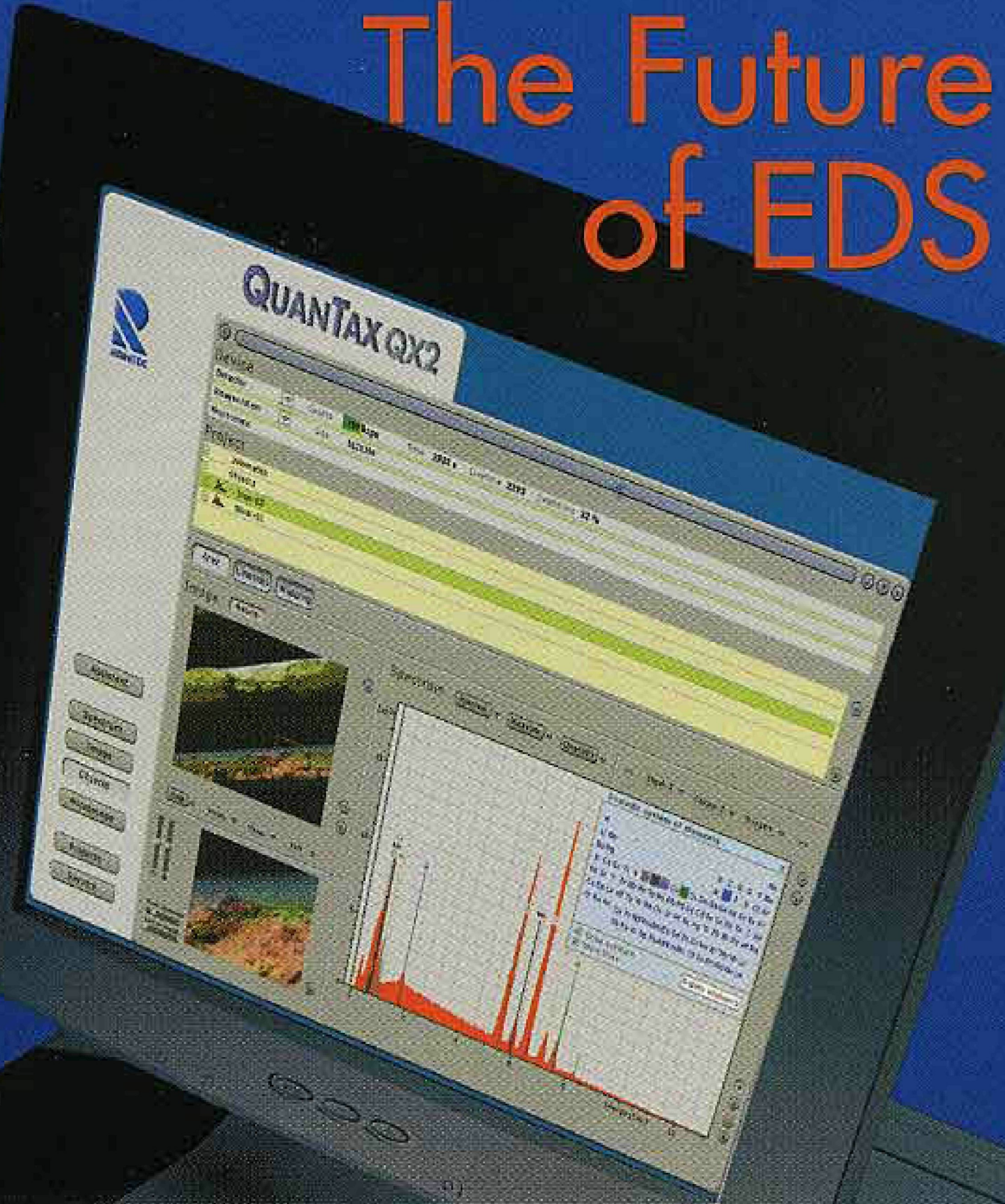

Highly precise analysis of all sample surfaces

$\mathrm{LN}_{2}$-free XFlash ${ }^{\oplus}$ Detectors (3rd Generation) now with light element detection

World's best resolution of $127 \mathrm{eV}$

Brilliant compositional ColorScans within seconds Fastest element mapping system available

The worlds rastest and

eosiest and yzer is here now.

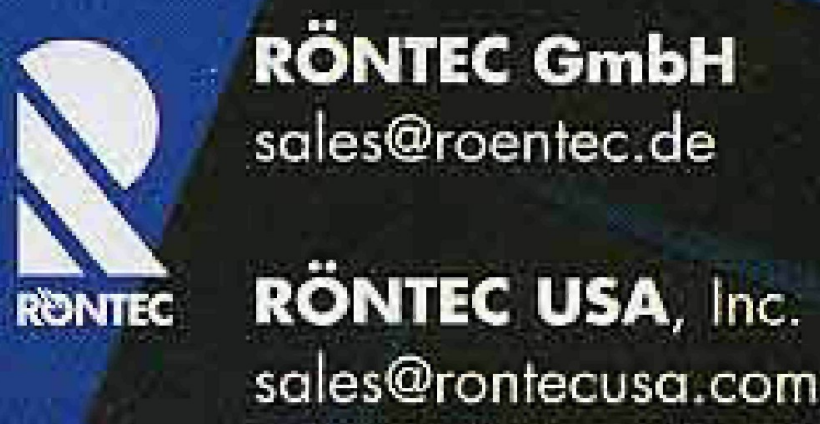

RöNTIEC France S.A.R.L. roentedrance@aol.com

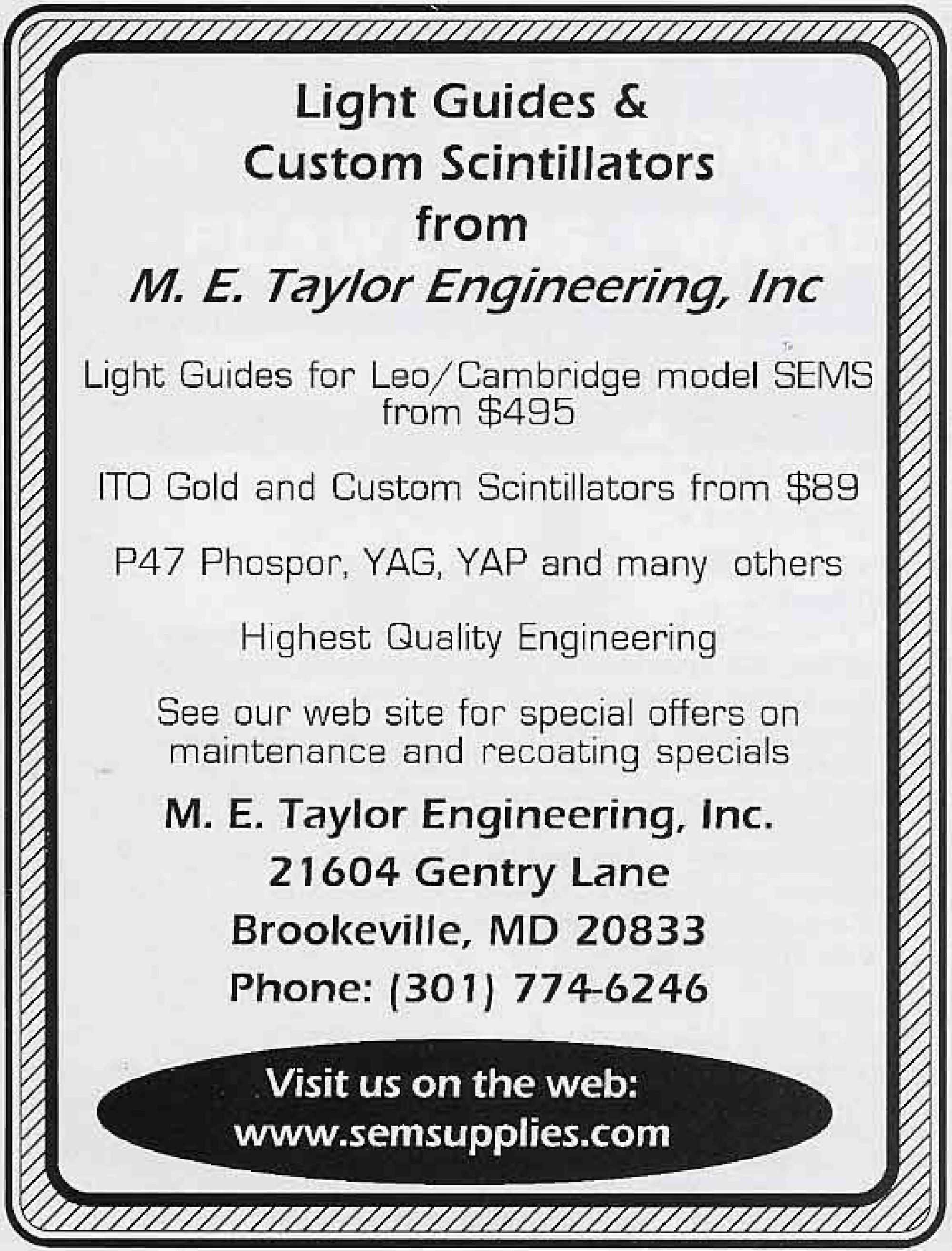

SERPETRENIX

PHONE/FAX

Introducing the MaxView Plus Digital Camera Attachment System

The MaxView Plus kit includes adapters to fit microscopes \& optical devices with any of the following attachments C-Mount, C/S-Mount, T-Mount, $23 \mathrm{~mm}$ Eyepiece Port, $30 \mathrm{~mm}$ Eyepiece Port, and 1.25" Eyepiece Ports

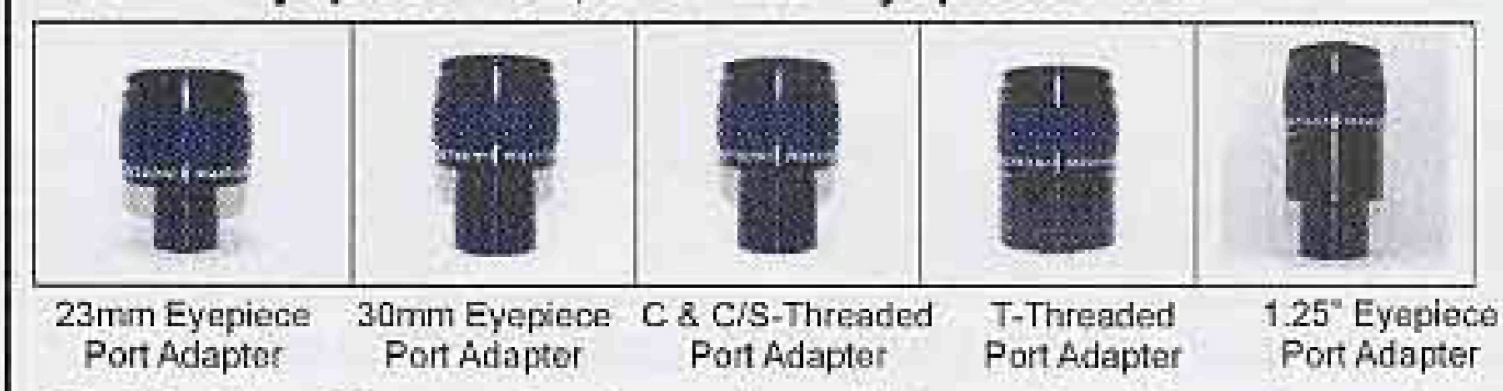

The MaxView Plus can also be used as a high quality wide angle eyepiece with the included thread-on eyeguard. The MaxView Plus is " $T$ " threaded on top so you will need the appropriate Attachment Kit to mate it to your particular digital camera. We carry dozens of attachment kits to fit most all popular digital cameras and we update them regularly so your MaxView Plus will not become obsolete when it is time to upgrade to a new camera If using it with a $35 \mathrm{~mm}$ SLR you will need the appropriate T-Ring for your brand of camera.

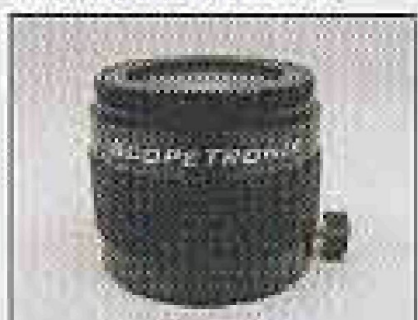

The heart of the Maxview system. A wide field $40 \mathrm{~mm}$ focal length four element symmetrical (Plossl) lens assembly specially mounted in a carefully designed custom cell. The lens is mounted only $1 \mathrm{~mm}$ from the top to maintain the close coupling required to reduce vignetting The cell features a unique sliding collar vignetting The cell features a unique sliding collar

it allows quick and easy removal of the camera, it allows you to adjust eyeguard height for easy visual use, allows you to adjust magnification when used with a $35 \mathrm{~mm}$ camera, and allows you to adjust lens position

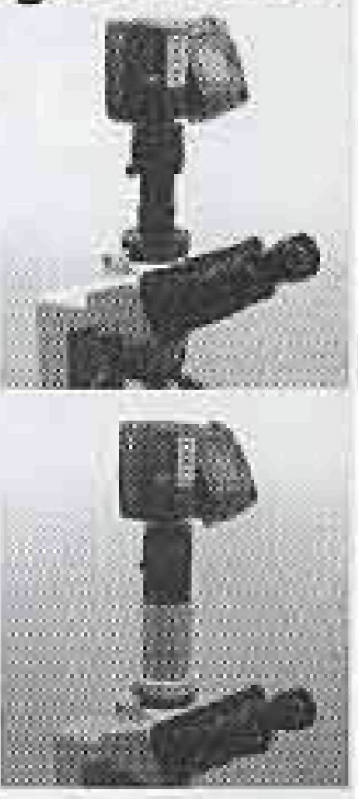
to minimize vignetting with a digital camera.

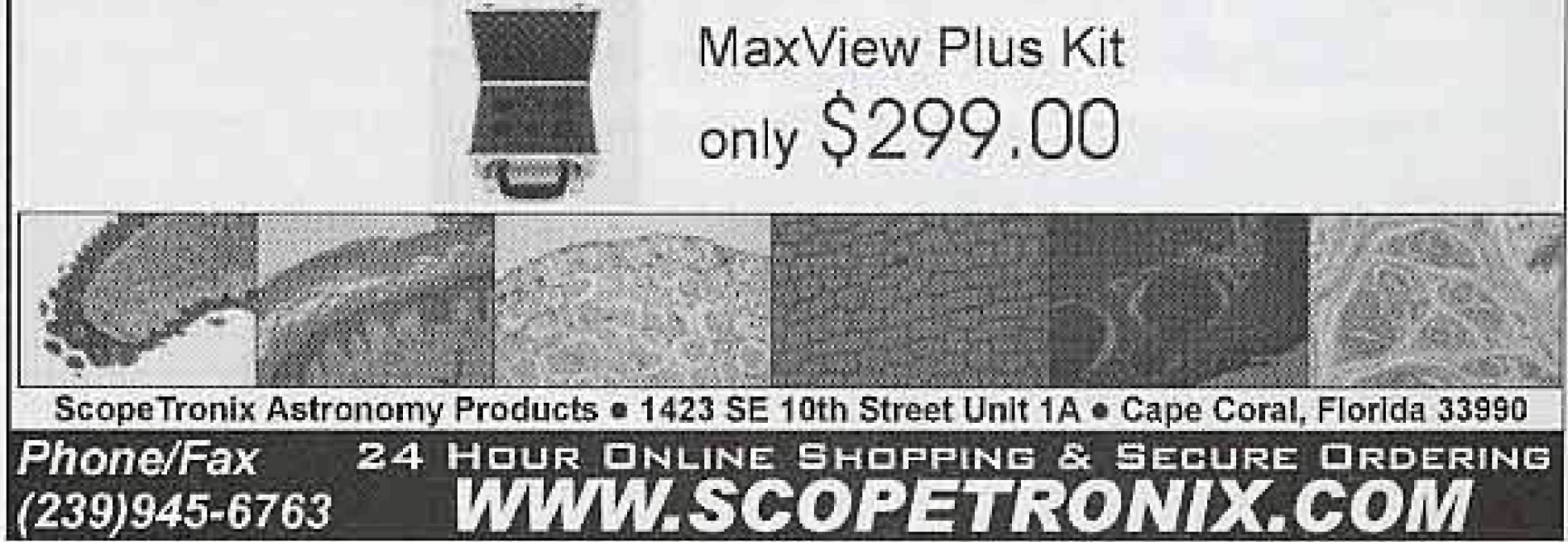




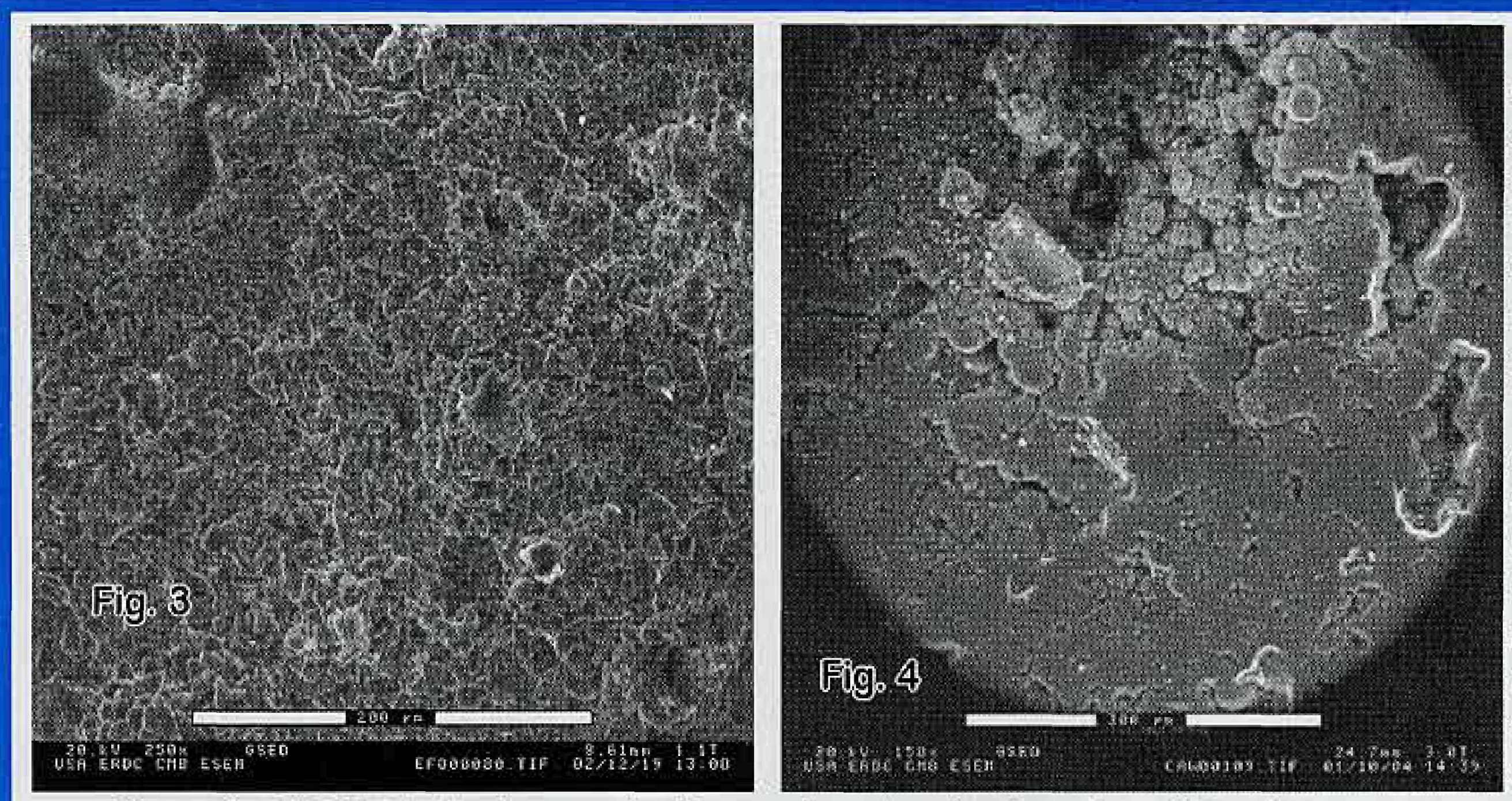

The first deformation map (Figure 5) mostly shows white space indicating none or very little deformation over the majority of the area. What is apparent is the light rarefactions (quite unorganized at this load) extending from the area of the razor sharp crack downwards and to the left. These unorganized rarefactions represent tensile microcracking in the area in front of the truly cracked portion of the beam. The dark red rarefactions and dark blue compactions at the bottom are areas where the DMT was trying to analyze the side of the gun column and failed to make proper comparisons. They should be ignored here. Figure 6 is the deformation map comparing no load and $6.0 \mathrm{lbf}$ load. In this deformation map, the crack has become organized at least in the top half of the map. At the bottom of the map, the deformation still represents disorganized cracking. Figure 7 shows the

Figure 3. ESEM Photomicrograph of cement paste after 3 cycles of freezing.

Figure 4. Photomicrograph of cement paste beam at $11 \mathrm{lbf}$ load

in this area after one cycle of freezing, but much greater damage is depicted after three cycles. This possibly is deformation caused by freezing and thawing around an entrapped air bubble under the surface of the imaged paste.

A further application of this technology has been applied to documenting deformation in cement paste in the region in front of a propagating crack. Cement paste is a quasi-brittle material that undergoes microcracking damage before it fails and actually produces a visible crack. The ESEM can be used in

Close observation of figure 4 confirms this view.

deformation map between no load and $11.0 \mathrm{lbf}$ that is also the image in figure 4 . In this map, the crack is now a full crack throughout the view of the ESEM.

These two examples show the capability of the DMT to document deformation that otherwise could not be seen easily in an ESEM image. The ability to compare pairs of images at different times and show the deformation that takes place gives the microscopist a new tool to analyze microscopic movement in samples viewed in the ESEM.

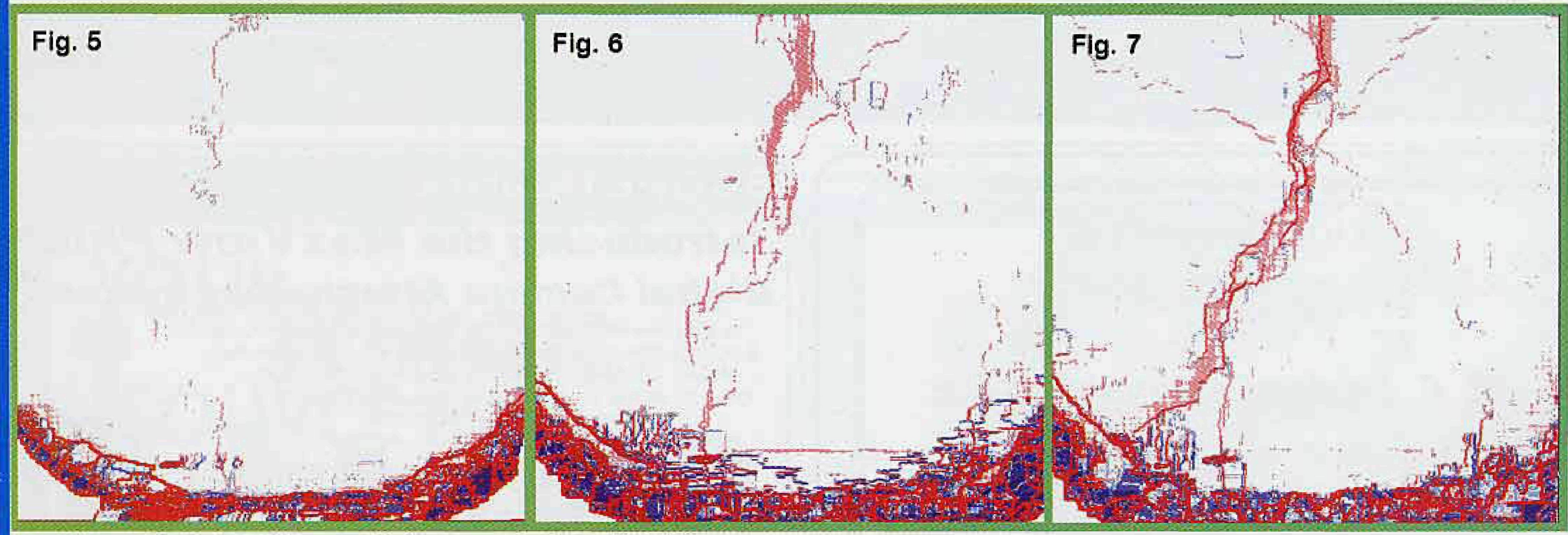

Figure 5. Deformation map between no load and $1.8 \mathrm{lbf}$.

Figure 6. Deformation map between no load and $6.0 \mathrm{lbf}$.

Figure 7. Deformation map between no load and $11.0 \mathrm{lbf}$.

conjunction with an internal loading stage to load a cement paste micro-beam with a sharp crack cast into the specimen. By focusing the ESEM at the tip of the sharp crack and recording images under increasing amounts of load, the progress of the microcrack deformations in the area in front of a crack tip can be documented before they become part of the crack. The ability to view this damage helps engineers understand the mechanisms that cause the damage. The DMT was used to track the growth of a crack in a cement paste beam loaded in the ESEM with a Fullum loading stage. Figure 4 is an ESEM photomicrograph of the area just in front of a sharp crack formed from the edge of a razor blade. The magnification for this experiment was set as low as possible to include the greatest amount of area in front of the sharp crack (the very dark area at the top center in Figure 4). The rounded black area at the bottom of the photomicrograph is the wall of the gun column. An image of the area was taken before any load was placed on the beam and load was applied in increments with photomicrographs taken at each increment. Figures 5,6 , and 7 are deformation maps representing DMT analysis between the before loading image and after the load increments of $1.8,6.0$, and $11.0 \mathrm{lbf}$.

\section{References:}

1. $X_{i}, Y_{\text {, }}$ Bergstrom, T. B., and Jennings, H. M., "Image intensity matching technique: application to the environmental scanning electron microscope," Computational Materials Science, vol. 2, 1994, pp 249-260.

2. Chen. J.J., Rothstein, D., Thomas, J.J., \& Jennings, H.M. "Application of the Deformation Mapping Technique to Assess Deterioration in Cement-Based Materials," Transportation Research Record, Journal of The Transportation Research Board, No. 1775, Concrete 2001, 28-35 (2001).

3. Neubauer, C. M., and Jennings, H. M., "The Use of Digital Images to Determine Deformation Throughout a Microstructure, Part II: Application to Cement Throughout a Microstructure," Journal of Materials Science, Vol $35,5751-5765(2000)$.

Disclaimer. The views expressed in this paper are the views of the writer, who is responsible for the facts and accuracy of the data. The contents do not necessarily reflect the official views or policies of the Engineer Research and Development Center, the Departments of the Arrmy or Air Force, or the Department of Defense. This paper does not constitute a standard, specification, or regulation. The contents of this paper are not to be used for advertising, publication, or promotional purposes. Citation of trade names does not constitute an official endorsement or approval of the use of such commercial products. 